\title{
Topology and Evolution of Technology Innovation Networks
}

\author{
Sergi Valverde ${ }^{1}$, Ricard V. Solé ${ }^{1,2}$, Mark A. Bedau ${ }^{3,4}$ and Norman Packard ${ }^{2,4}$ \\ ${ }^{1}$ ICREA-Complex Systems Lab, Universitat Pompeu Fabra, Dr. Aiguader 80, 08003 Barcelona, Spain \\ ${ }^{2}$ Santa Fe Institute, 1399 Hyde Park Road, New Mexico 87501, USA \\ ${ }^{3}$ Reed College, 3203 SE Woodstock Blvd., Portland 97202, USA \\ ${ }^{4}$ ProtoLife, Parco Vega, Via della Liberta 12, Marghera 30175, Venice, Italy
}

\begin{abstract}
The web of relations linking technological innovation can be fairly described in terms of patent citations. The resulting patent citation network provides a picture of the large-scale organization of innovations and its time evolution. Here we study the patterns of change of patents registered by the US Patent and Trademark Office (USPTO). We show that the scaling behavior exhibited by this network is consistent with a preferential attachment mechanism together with a Weibullshaped aging term. Such attachment kernel is shared by scientific citation networks, thus indicating an universal type of mechanism linking ideas and designs and their evolution. The implications for evolutionary theory of innovation are discussed.
\end{abstract}

PACS numbers: $87.23 . \mathrm{Kg}$

\section{INTRODUCTION}

Innovation takes place both in nature and technology [1]. Either through symbiosis [2], tinkering [3] or design [4, 5] new functional structures and artifacts are obtained. Such new entities often result from the combination of predefined designs or building blocks, although a completely new solution can also emerge. This is the case for example of the replacement of vacuum tube technology by semiconductors. However, the majority of technological (and evolutionary) changes take place by means of a progressive path of change. Such steady and successful transformation of designs is largely based on an extensive combination and refinement of existing designs.

A surrogate of the ways in which innovations take place in time is provided by patent files. Patents are welldefined objects introducing a novel design, method or solution for a given problem or set of problems. Additionally, they indicate what previous novelties have been required to build the new one. In order to gain insight into the global organization of the patterns of innovation and their evolution in technology, here we study a very large data base including all USPTO patents from 1975 to 2005 [6].

As it occurs with the fossil record for evolution, the record of patents through time provides us with the opportunity of seeing how new inventions emerge and how they relate to previous ones. A given patent will typically require new solutions and previously achieved results. Looking at how patents link to each other is the simplest way of having a large scale picture of the patterns and processes associated to the collective dynamics of innovation unfolds [7, 8]. Many interesting questions can be formulated in relation to this: what is the global organization of interactions among innovations? Is this a repeatable pattern? How are similar classes of innovations related among them? Do these patterns respond to history-dependent rules or are instead describable by means of simple models? These questions are addressed here and it is shown that a standard statisti- a

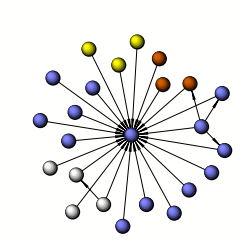

b

d
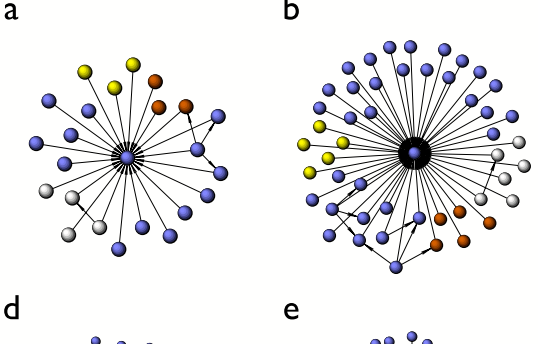

e

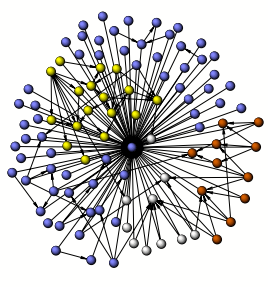

FIG. 1: From (a) to (f), evolution of a patent subset related to computed tomography. The hub in the center corresponds to the precursor invention by G. Hounsfield (US patent 3778614).

cal physics approach provides a good picture of how these webs emerge.

The paper is organized as follows: in section II the data set analysed is presented. In section III the topological trends exhibited by the patent citation network are discussed under the light of a model of graph growth with aging (section IV). In section V our basic results are summarized and its implications outlined.

\section{PATENT CITATION NETWORKS}

Previous studies have measured the value of an innovation by means of the analysis of patent citations, i.e., the rate of receiving new citations. However, innovation is an elusive notion that is difficult to measure properly 
and existing measures provide limited insight [7]. It is a difficult task to find useful indicators for the value of innovations. In this context, we introduce patent citation networks as an appropriate approach to the global analysis of the process of technological innovation. Recent work in complex networks provides several models that describe or reproduce structural features of natural and artificial evolving systems. Here, we will show how innovation can be described as a process of network growth following some specific rules. In particular, our model provides a rigorous statistical test to assess the balance between patent importance and patent age, i.e., Price's "immediacy factor" 7].

The set of patents and their citations describes a (socalled) patent citation network $G$. The patent network belongs to the general class of citation networks, which includes the scientific citation network. Here, nodes $v_{i} \in G$ represent individual patents and the directed link $\left(v_{i}, v_{j}\right)$ indicates that patent $v_{i}$ is a descendent of patent $v_{j}$. In order to illustrate the power of the network approach, we have re-analyzed the evolution of a well-know patent dataset. Figure 1 shows the time evolution for the subset of patents in Computer Tomography (CT), from 1973 to 2004 . A smaller subset of this dataset was analysed in [13]. The figure indicates that some patents receive much more citations than others. In particular, the hub at the center corresponds to the very first patent in CT associated with its invention by G. Hounsfield.

Interestingly, the network analysis reveals some other patterns that cannot be easily recovered by other means. For instance, in figure 1 we can appreciate the modular organization of the CT patents. Here we have used Clauset et al. algorithm 14 to detect community structure in large networks. Roughly speaking, topological modules are defined as groups of nodes having more links among them than with other elements in the graph. Thus, patents belonging to the same module share a common color. Although we have not explored this problem in detail, direct inspection of the networks shown in figure 1 reveals that the modular structure seems to correlate well with shared functional traits. As an example, the white module involves several related patents associated with X-ray tomography.

Beyond specific patterns of patent evolution, here we aim to detect universal trends in the global evolution of the US patent system. The patent citation network (PCN) analyzed here has $N=2801167$ nodes and $L=$ 18053661 links. Its time evolution from 1976 to 2005 is shown in figure2 The number of patents at a given time $t$ scales as a power law:

$$
N(t) \sim t^{\theta}
$$

with an exponent $\theta=1.45 \pm 0.06$. Some recent papers have explored the patent citation datasets at different levels, including a graph theoretical approach on a large scale [9] or involving a more specific case study, such as fuel cell research [10]. Here we will show that the statisti-

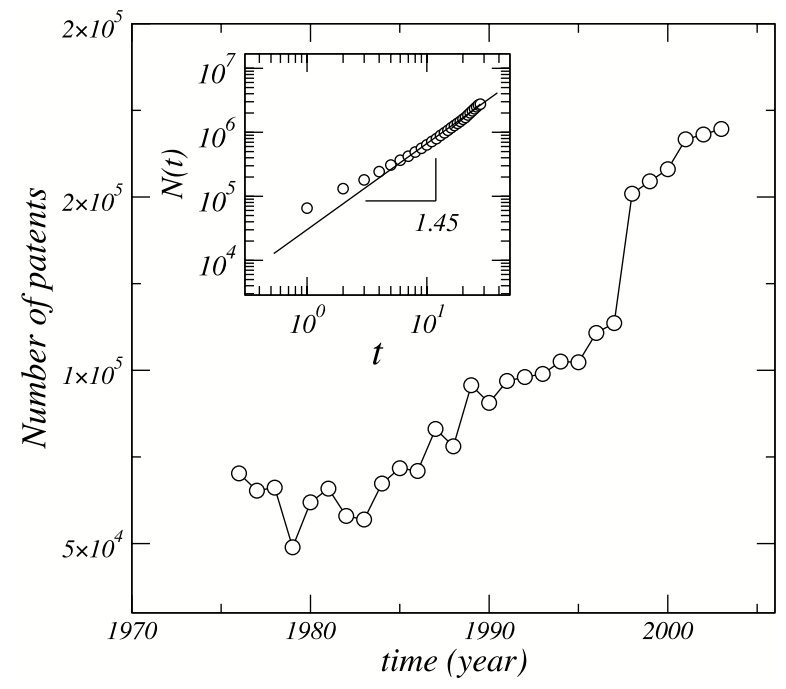

FIG. 2: Time evolution of the number of patents $N(t)$ in the USPTO dataset from 1973 to 2004. Inset: Cumulative number of patents on a log-log scale, showing a scaling $N(t) \sim$ $t^{\theta}$

cal features of this network can be explained by using an appropriate attachment kernel describing how successful patents become more linked and how this preferential attachment decays with age.

\section{DISTRIBUTION OF PATENT CITATIONS}

Citations are often interpreted as indicators of innovation size or economic value [? ]. The distribution of innovation size (defined as the number of citations to a patent) is skewed [8, 11, 12]. However, there is an ongoing discussion about the particular nature of this distribution. In particular, there is no general agreement whether it follows a log-normal or Pareto distribution [12, 15]. Still, there are common patterns like the existence of some extreme values, which is consistent with a power-law tail. We report similar features in the indegree distribution studied here (see below).

The in-degree distribution $P_{i}(k)$ is equivalent to the so-called distribution of number of patent citations. Figure 3A shows the in-degree distribution for the patent citation network in 2004. Notice that $P_{i}(k)$ is neither exponential nor a simple power law. Instead, we have found that an extended power-law form fits the in-degree distribution very well:

$$
P_{i}(k) \sim\left(k+k_{0}\right)^{-\gamma}
$$

where $k_{0}=19.46 \pm 0.22$ and $\gamma=4.55 \pm 0.04$. This extended power-law reduces to a power-law when $k \gg k_{0}$ and it degenerates to an exponential distribution for $k \ll k_{0}$. The extended power-law distribution has been 
related to a mixed attachment mechanism [18]. However, here we will show that this explanation does not apply for the patent citation network. Instead, we propose that the extended power-law form for the in-degree distribution stems from a combination of both preferential attachement and aging [19].

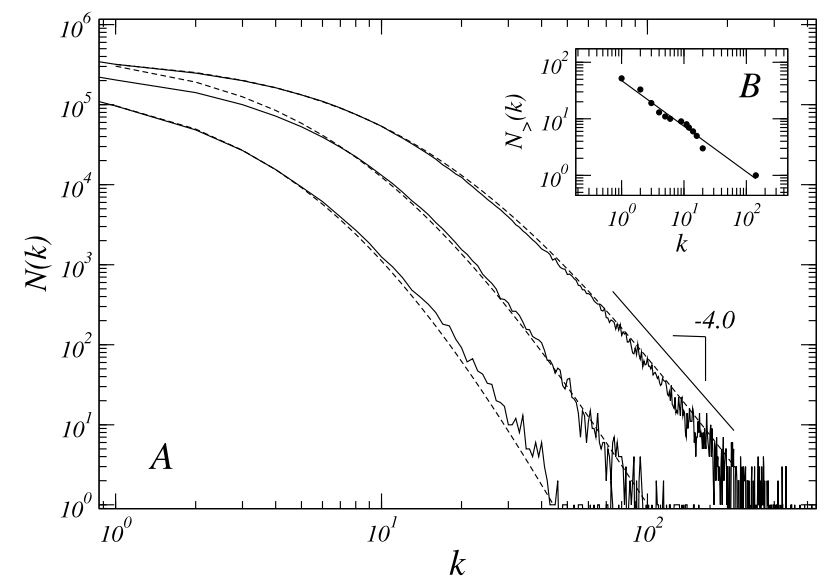

FIG. 3: (A) The in-degree distribution for the patent citation network follows an extended power-law distribution, $P_{i}(k) \sim\left(k+k_{0}\right)^{-\gamma}$. Three distributions are displayed for three different time windows, namely 1984 (leftmost), 1992 (center) and 2002 (rightmost). (B) The in-degree distribution for the subset of patents displayed in fig. $1 \mathrm{f}$ (for computer tomography) is roughly approximated by a scale free distribution. The leftmost point indicates the central hub in fig.1.

\section{EVOLUTION}

Lets us assume that every patent has a unique identifier $0<i<t$. Our model starts at time $t=0$ when there is only one patent in the network. From this initial network, we add a new patent $i$ at every time step that references $m$ previous patents. Two main forces drive the evolution of the patent citation network. First, it is natural to assume that the number of patent citations (i.e., incoming links) is a surrogate of its relevance [? ]. Useful patents are more likely to receive further citations than marginal inventions. Thus, the probability of receiving new citations should be proportional to the current number of citations. This rule parallels the preferential attachment mechanism of network growth [20]. Under this rule new elements entering the system connect with other nodes with a probability $\Pi(k)$ that is proportional to its degree, i.e.,

$$
\Pi(k) \sim k
$$

However, old patents tend to be less relevant in the context of recent innovations: attachment rates decay as the patent losses value. In particular, patents are released to the public domain after some finite period of exploitation.
The evolution of complex networks involving both preferential attachment and aging has been extensively studied. In particular, Dorogovtsev and Mendes (DM) determined analytically the scaling properties of the resulting networks [19]. In the DM model, the rule of attachment scales now as:

$$
\Pi(k, \tau) \sim k \tau^{-\alpha}
$$

where $\tau=t-i$ indicates the age of the $i-t h$ node and the exponent $\alpha$ (which is positive) weights how fast is the aging affecting the likelihood of attachment. Extensions of this attachment probability kernel include accelerated growth with $\Pi(k, \tau) \sim k^{\beta} \tau^{-\alpha}$ and exponential aging kernel $\Pi(k, \tau) \sim k \exp \left(-\tau^{\alpha}\right)[18$ ].

Finally, some models of scientific citation networks take into account the simultaneous evolution of author and paper networks [21]. In these models, the rule of attachment behaves as:

$$
\Pi\left(k_{i}, \tau\right) \sim k_{i}^{\beta} \tau^{\alpha-1} e^{-\left(\frac{\tau}{\tau_{0}}\right)^{\alpha}}
$$

when the time-dependent component follows a Weibull form. Here, $\tau_{0}$ controls the rightward extension of the Weibull curve. As $\tau_{0}$ increases, so does the probability of citing older papers.On the other hand, small values of $\tau_{0}$ indicate strong aging that favors recently published patents [21]. Here we choose the simplest assumption (preferential attachment $\beta=1$ ) and consider the aging function in eq. 5. Consequently, the average connectivity of the $i-t h$ patent at the time $t$ evolves according to the following equation:

$$
\frac{\partial \bar{k}(i, t)}{\partial t}=\frac{m \bar{k}(i, t) f(t-i)}{\int_{0}^{t} \bar{k}(u, t) f(t-u) d u}
$$

where $m$ is the number of links introduced at each step ( $m=1$ is the DM model). Now we address the following question: is the above equation consistent with the patent network evolution? In the following, we will estimate the form of the attachment kernel (and the corresponding $\alpha, \beta$ and $\tau_{0}$ parameters) for the patent citation data.

First, we consider system size $N$ as our time index instead of real time $t$. This way we avoid any bias due to the pattern of non-linear growth $(1)$ and attach to the standard formulation of network models. Then, eq. (6) becomes:

$$
\frac{\partial \bar{k}(i, N)}{\partial N}=\frac{m \bar{k}(i, N) f(N-i)}{\int_{0}^{t} \bar{k}(u, t) f(N-u) d u}
$$

Using $\partial \bar{k} / \partial N=(\partial \bar{k} / \partial t)(\partial t / \partial N)$ and the timedependent scaling $N(t)=A t^{\theta}$, we have: 


$$
\left.\frac{\partial \bar{k}(i, N)}{\partial N}=\left(\frac{1}{\theta} N(t)-A\right)^{\frac{1}{\theta}-1}\right) \frac{\partial \bar{k}(i, t)}{\partial t}
$$

Now the whole time interval $N$ is partitioned into $N / \Delta N$ time slots comprising the same number $\Delta N \ll N$ of patents. Here, $N \approx 2.8$ million patents corresponding to the time interval 1976-2005. The $s-t h$ time slot has the same number of new $\Delta N=10^{5}$ patents.

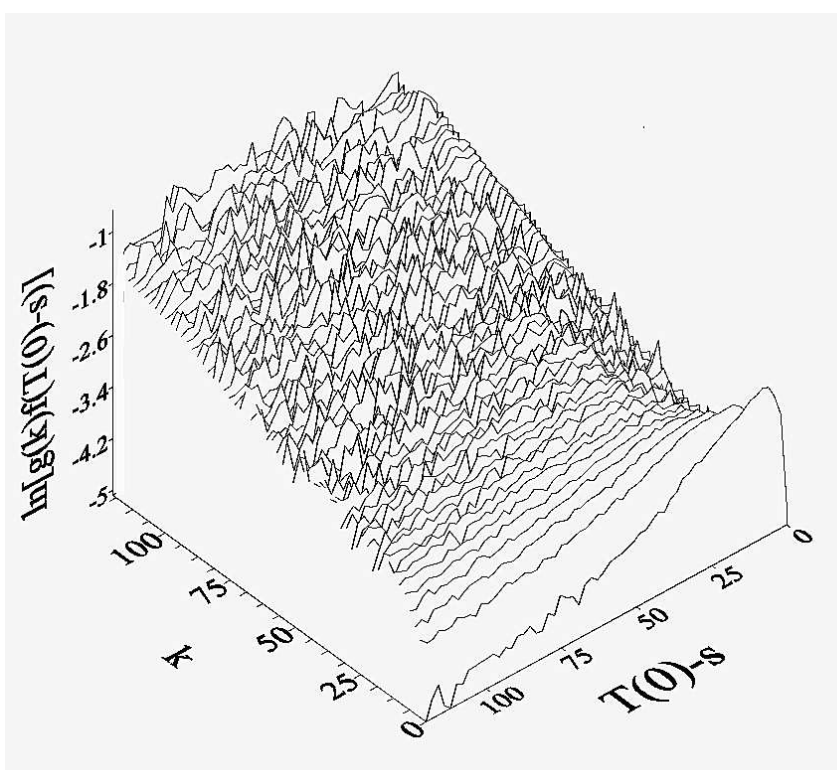

FIG. 4: The normalized attachment kernel $\Pi(k, \tau) \sim g(k) f(\tau)$ determined numerically for the patent citation network at $T_{0}=2002$.

To measure the attachment rule $\Pi\left(k_{i}, \tau\right)$ we monitor to which old patent new patents link, as a function of in-degree $k_{i}$ and age $\tau$ (recall that here $\tau$ is measured in number of time slots). We have assumed that attachment is the product of a preferential attachment function $g(k)$ and an aging function $f(\tau)$ :

$$
\Pi(k, \tau) \sim g(k) f(\tau)
$$

Following [22], we study the citation process in a relatively short time frame (a time slot $\Delta N)$. The large number of nodes in the system (in the order of $10^{6}$ nodes) ensures that we will gather sufficient samples to recover the attachment kernel. We divide the evolution of the system in three stages: (i) the system before slot $T_{0}$, (ii) the system between slots $T_{0}$ and $T_{1}=T_{0}+1$ and (iii) the system after $T_{1}$. When a $T_{1}$ node joins the system we record the age $\tau$ and the in-degree $k$ of the $T_{0}$ node to which the new node links. We count all the citations made by new nodes between $T_{1}$ and $T_{1}+1$. The number of citations received by nodes $T_{0}$ from $T_{1}$ nodes normalized by the in-degree frequency $P(k)$ is an approximation to the attachment kernel (see fig. 44).
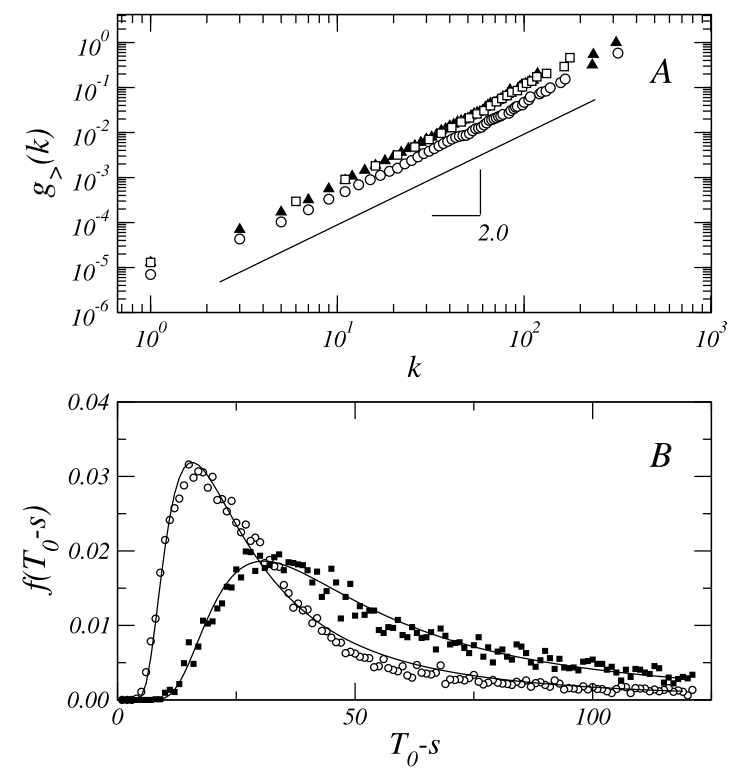

FIG. 5: Estimation of the attachment rule for the patent citation network at $T_{1}=2003$. (a) Preferential attachment function fits a scaling-law $g_{>}(k) \sim k^{\beta+1}$ with $\beta=1$. Each curve corresponds to nodes having the same age. (b) Fitting for the aging function $f(\tau)$ predicts the Weibull distribution described in the text with $\alpha \approx-1.45$. Each curve corresponds to nodes having the same in-degree ( $k=1$ for white balls and $k=5$ for solid balls). For every curve we have used $T_{0}=T_{1}-1$.

Using our dataset, we have estimated that $g(k) \sim k^{\beta}$ and found $\beta=1$, which further validates our assumption of preferential attachment (see fig. [5A). Notice that in our fittings we have used the cumulative function $g_{>}(k)=\int_{0}^{k} g(k) d k$ to reduce the noise level. On the other hand, fig. 5B shows the Weibull distribution, which fits very well the aging function $f(\tau)$ :

$$
f(\tau) \sim \alpha \tau_{0}^{-\alpha} \tau^{\alpha-1} e^{-\left(\frac{\tau}{\tau_{0}}\right)^{\alpha}}
$$

with an exponent $\alpha \approx-1.45$ and $\tau_{0} \approx 40$. An obvious advantage of using the Weibull form is that in naturally includes as limit cases both exponential and Gaussian distributions.

The common structure of the aging term found here and in the network of paper citations 21] suggests that common patterns of organization and evolution might be shared. The paper citation graph, obtained by looking at the list of references included in each paper, is in fact close to the basic rules defining the patent citation graph. In both cases, cross-links are associated to some underlying set of features which are shared by patents or papers. As it occurs with the patent case, new papers are based on previous ones providing the background required to build a new idea. On the other hand, as new ideas and concepts develop into well-defined areas, they will tend to attach less to more generic or older works. 
Additionally, the observed modular organization which might also contribute to deviate from the simple powerlaw attachment assumed in previous theoretical studies. What seems clear is that there might be some universal trends canalizing the growth of innovation networks, whether scientific or technologic.

\section{DISCUSSION}

The patterns of innovation emerging in our society are the outcome of an extensive exchange of shared information linked with the capacity of inventors to combine and improve previous designs. Even very original inventions are not isolated from previous achievements. A patent can be identified as an object which needs a minimum amount of originality to be considered as truly different from previous patents. Moreover, to be obtained, it must properly refer to related patents in a fair way. Such constraints make this system specially interesting since we can wisely conjecture that it represents the expansion of real designs through some underlying technology landscape. These designs can be just small improvements or large advances. Our analysis provides a quantitative approach to this evolving structure using the approach of statistical physics.

We have shown that the underlying rules of network change for our system reveal a mixture of preferential attachment favouring a rich-gets-richer mechanism together with an aging term weighting the likelihood of citing old patents. As the network grows, recent patents will tend to cite recent designs (since innovation is likely to involve redefining recent inventions) and be less likely to link to old patents. The consequence of this, as predicted by previous mean field models (refs) is that the expected scaling law in the degree distribution associated to pref- erential attachment kernels will be modified in significant ways. Here we have shown that the network of patents, defined by using the indegree as a surrogate of patent relevance, scales as $P(k) \sim\left(k+k_{0}\right)^{-\gamma}$ with $\gamma>4$. This is not far from previous predicted scaling laws (DM) associated to preferential attachment and power law aging (i. e. $f(t) \sim t^{-\alpha}$ which predict $P(k) \sim k^{-\gamma(\alpha)}$ (with $\gamma \sim 4$ for $\alpha \sim 0.5)$. However, the humped shape of our aging term (as described by the Weibull distribution) makes necessary to modify these approximations.

As a final point in our discussion, it is worth noting that we have strong correlations among patents indicating a complex organization in modules. As shown by the example in figure 1, together with the nonlinearities associated with the attachment rules, there is some underlying community structure in the patent network that deserves further exploration. The emergence of modules is a natural consequence of the specialized features shared by related patents. But it might also reveal the structure of the innovation landscape itself: new patents related to previous ones can also be understood as improved solutions that explore the neighborhood of previous solutions. This view would provide a quantitative picture of the topology of technology landscapes [23, 24]. Such an evolutionary interpretation in terms of fitness functions will be explored elsewhere.

\section{Acknowledgments}

We thank Vincent Anton and Marti Rosas-Casals for useful discussions. This work has been supported by grants FIS2004-05422, by the EU within the 6th Framework Program under contract 001907 (DELIS), by the James McDonnell Foundation and by the Santa Fe Institute.
[1] Erwin, D. H. and Krakauer, D. Science 304, 1117 (2004)

[2] Margulis, L. and Fester, R. (eds.), Symbiosis as a source of evolutionary innovation. MIT Press (1991).

[3] Jacob F., Science 196, 1161 (1977).

[4] Solé, R.V., Ferrer-Cancho, R., Montoya, J. M. and Valverde, S., Complexity, 8, 20 (2002).

[5] Lienhard, J. H. How invention begins. Oxford U. Press (2006).

[6] US Patent and Trademark Office (http://www.uspto.gov/)

[7] Jaffe, A. B., and Trajtenberg, M., Patents, Citations and Innovations: A Window on the Knowledge Economy, MIT Press (2002).

[8] Kuznets, S., in Nelson, R. R., (eds), The Rate and Direction of Incentive Activity: Economic and Social Factors, Princeton U. Press (1962).

[9] Csardi, G. et al., http://arxiv.org/abs/physics/0508132

[10] Verspagen, B. Eindhoven Center for Innovation Studies, Working Paper 05.11 (2005).

[11] Scherer, F. M., Am. Econ. Rev., 55, 1097 (1965).
[12] Scherer, F. M., Ann. d'Economie Stat. 49/50, 495 (1998).

[13] Trajtenberg, M., RAND Journal of Economics, 21(1), 172 (1990).

[14] Clauset, A., Newman, M. E. J. and Moore, C. Phys. Rev. E70 066111 (2004).

[15] Harhoff, D., Scherer, F.M., and Vopel, K., in Grandstrand, O., (ed.) The Economics of the Patent System, Routledge (2003).

[16] Dorogovtsev, S. N. \& Mendes, J. F. F., Evolution of Networks: From Biological Nets to the Internet and $W W W$, Oxford University Press, New York (2003).

[17] Ball, P., Critical Mass: How one thing leads to another, Arrow Books, (2004).

[18] Shao, Z.-G., Zou, X.-W., Tan, Z.-J., and Jin, Z.-Z., J. Phys. A: Math. Gen. 39, 2035 (2006).

[19] Dorogovtsev, S.N., and Mendes, J. F. F., Phys. Rev. E. 62, 1842 (2000).

[20] Barabasi, A.-L., and Albert, R., Science, 286, 509 (1999).

[21] Borner, K., Maru, J. T., and Goldstone, R. L., PNAS, 
101, 5266 (2004).

[22] Jeong, H., Néda, and Barabási, Europhys. Lett. 61, 567 (2003).

[23] Kauffman, S. A. At home in the universe. Oxford U.
Press (1995).

[24] Kauffman, S. A. Investigations. Oxford U. Press (2000). 